Gynäk. Rdsch. 1967;4:I-VIII

\title{
Contents, Vol. 4, 1967
}

Übersichtsaufsatz Tapfer, S.: Wandel der Pathologie und geburtshilflichen Bedeutungdes Beckens 241

\section{KURZFASSUNGEN VON ZeITSCHRIFTENARTIKELN}

Abdel Naby, S.: vide Talaat, $M$.

Acheson, E. D.: vide Hobbs, M. S. T.

Alison, F.: vide Desmonts, G.

Anderson, W. A. D. und Gunn, S. A.: Kritische Überprüfung der Scheidenspülung als Suchmethode zur Zervixkrebserkennung Apostolakis, M.; Becker, H. und Voigt, K. D.: Der Einfluß der Gabe von Lynestrenol auf die Ausscheidung von Testosteron, Östrogenen, Pregnandiol und Gesamtgonadotropinen während des menstruellen Zyklus

Barbo, Dorothy M.: vide Hofmeister, F. J.

Barnes, M. L.: vide Gray, L. A.

Baudelot, J.: vide Desmonts, G.

Becker, H.: vide Apostolakis, M.

Bergman, P.; Kjellander, J.; Thunell, G. und Zettergren, L.: Listeriose und Gravidität

Bericht des Komitees für öffentliche Gesundheit. Medizinische Akademie New York: Das Wiederaufleben der Geschlechtskrankheiten

Bierme, R.: vide Ducos, J.

Bishop, M. W. H.: vide Lyster, W. R.

Blank, B. und Mirgone, O. R.: Nachweis der Genitaltuberkulose bei der Autopsie von Frauen mit Lungentuberkulosen

Brest, A. N.; Heider, C.; Mehbod, H. und Onesti, G.: Medikamentöse Behandlung der durch Diuretika ausgelösten Hyperurikämie

Caplan, R. M.: Die Reizwirkung des Stuhles bei der Entstehung des Pruritus ani Garrig, Shirley: vide Kaufman, R. H.

Cavenagh, A. J. M.: Die Bedeutung des allgemein-ärztlichen Entbindungsheims Chapus, G.: vide Jaulmes, B.

Chayon, E.: vide Ducos, J.

Craig, J. M.: vide Kistner, R. W.

Committee on Maternal Welfare: Studien über die Müttersterblichkeit: Lungenembolie

Committee on Maternal Welfare: Studien über die Müttersterblichkeit: Uterusruptur Committee on Maternal Welfare: Studien über die Müttersterblichkeit: Die post-partum-Blutung Couvreur, J.: vide Desmonts, G. Denys, P.: vide Lauweryns, J.-M. 8661 
IV

Inhaltsverzeichnis

Desmonts, G.; Couvreur, J.; Alison, F.; Baudelot, J.; Gerberaux, J. und Lelong, M.: Epidemiologische Studie über die Toxoplasmose: Über den Einfluß des Kochens von Fleisch auf die Häufigkeit der Infektion beim Menschen 50

Donovan, J. C.: Die Beziehung zwischen Arzt und Patientin in der Gynäkologie 19

Ducos, J.; Bierme, R.; Montalegre, J.; Marty, Y. und Chayon, E.: Wie kann man durch die Injektion inkompatiblen Blutes bedingte Transfusionszwischenfälle verhindern? 37

Dunn, L.: vide Steingold, L.

Edwards, D. A. W.: vide Phillips, S. F.

Eggermont, E.: vide Lauweryns, J.-M.

Erickson, C. C.: vide Lundin, F. E., Jr.

Finger, Sally: Gruppentherapie mit ledigen Müttern und deren Eltern

Gal $\lambda$ mbos, L. und Scholz, Magda: Perinatale Mortalität mit besonderer Berücksichtigung von Entwicklungsstörungen 17

Gedda, L. und Milani-Comparetti, M.: Sind Zwillinge Frühgeburten ?

George, P.: vide Kreel, L.

Gerberaux, J.: vide Desmonts, G.

Geslin, P.: vide Jaulmes, B.

Gray, L. A. und Barnes, M. L.: Endometriose und Ovarialkarzinom 104

Gray, M. J.: vide Watanabe, $\lambda$.

Griffith, C. T.: vide Kistner, R. W.

Grumbach, M. M. und Kaplan, S. L.: Über plazentare Herkunft und Reinigung eines Ghorion «Wachstumshormon-Prolactin» und dessen immunologe Bestimmung in der Schwangerschaft 67

Guimbretière, J. und Lebeaupin, R.: ABO-Kontrolle vor der Transfusion am Krankenbett durch «Controlab»: Analyse einer ersten Serie von Resulta-

ten 41

Gunn, S. A.: vide Anderson, W. A. D.

Habib, Y. A.: vide Talaat, M.

Harris, J. M. und Warsaw, E.: Fettsucht, ein vielgestaltiges Problem: Beobachtungen über die Behandlung mit Choriongonadotropin als Ergänzung

diätetischer Maßnahmen 59

Hawksworth, E.: vide Steingold, L.

Heider, C.: vide Brest, A. N.

Hellinga, G.: Klassifizierung der männlichen Subfertilität als Anleitung für die Behandlung 218

Higazy, A. M.: vide Talaat, $M$. 
Hobbs, M. S. T. und Acheson, E. D.: Perinatale Sterblichkeit und die Organisa tion des geburtshilflichen Dienstes im Oxford Areal im Jahr $1962 \quad 1$ Hofmeister, F. J. und Barbo, Dorothy M.: Gibt es wirklich die Krebserkennung in der Sprechstunde? 84

Hopkinson, W. I.: vide Hutchinson, J. A.

Houde, R. W.: vide Schottenfeld, D.

Hutchinson, J. A.; Kerr, M. M.; Williams, K. G. und Hopkinson, W. I.:

Überdrucksauerstoff in der Wiederbelebung von Neugeborenen 139

Ibrahim, Z. A.: vide Talaat, M.

Jaulmes, B.; Terrier, E.; Chapus, G. und Geslin, P.: Das Risiko bei Trans-

fusionen in der Krankenhauspraxis $\quad 33$

Jindra, J.: vide Palisa, V.

John, A. H.: Der Einfluß der mütterlichen Hypoxie auf die Herzschlagfrequenz

des Kindes in utero 266

Johnson, F. L.: Erfahrungen mit der Margulies-Spirale $\quad 212$

Inhaltsverzeichnis $\mathrm{V}$

Jonas, E. G.: Der Wert pränataler Bettruhe bei Mehrlingsschwangerschaft 14 Josso, F.; Jungers,

P. und Trine, G.: Das Verhalten bei einem durch Blut-

unverträglichkeit bedingten Transfusionszwischenfall

43

Jungers, P.: vide Josso, F.

Kahr, E. und Schreyer, H.: Zur Frage der präoperativen Bestrahlung des

Mammakarzinoms im Stadium I und Ha 108

Kaplan, S. L.: vide Grumbach, M. M.

Kaufman, R. H.; Spjut, H. J. und Carrig, Shirley: Zervix-Scheiden-Abstrich

bei Teenagern 90

Kavanaugh, G.: vide Schirger, A.

Kerr, M. M.: vide Hutchinson, J. A.

Kind, H. und Schorno, O.: Grundsätzliches zur psychiatrischen Begutachtung der Schwangerschaftsfähigkeit 272

Kiss, G. und Steczik, A.: Todesfälle bei septischem Abort

Kistner, R. W.; Griffith, C. T. und Craig, J. M.: Die Anwendung von Gestagenen zur Beeinflussung des Korpuskarzinoms 281

Kjellander, J.: vide Bergman, P.

Knowles, Elizabeth: Erfahrungen mit oraler Kontrazeption auf den Fidji-

Inseln 23

Kolárová, J.: vide Palisa, V.

Kreel, L. und George, P.: Lymphangiographie nach Ablatio mammae. Nach-

weis von Metastasen und Ödem 294

Lacomme, M. und Lewin, D.: Die vorzeitige Entbindung wegen Hypertonie und

Albuminurie. Statistik der Klinik Baudelocque $\quad 112$

Larsson, J.: Listeriainfektionen in Schweden 129

Lauweryns, J.-M.; Eggermont, E.; Van den Driessche, A. und Denys, P.: Die

sekundäre Lungenatelektase der Neugeborenen mit hyalinen Membranen 230 Lebeaupin, R.:

vide Guimbretière, J. Lelong, M.: vide Desmonts, G. Lewin, D.: vide Lacomme, M. Limb, L.:

vide Steingold, L. Lowe, C. R.: vide Richards, I. D. G. 
Lubke, W. E.: Endometrium-Spülung. Suchmethode zur Krebserkennung .... 94 Lundin, F. E., Jr.; Erickson, C. C. und Sprunt, D. H.: Sozioökonomische Ver-

teilung des Zervixkarzinoms in Beziehung zur frühen Heirat und zur

Schwangerschaft 27

Lyster, W. R. und Bishop, M. W. H.: Eine Beziehung zwischen Regenfällen und der Geschlechtsverteilung beim Menschen 274

Magnin, R. und Seigneurin, R.: Kontrolle der ABO-Gruppe am Krankenbett

vor jeder Transfusion 40

Malek, A. Y.: vide Talaat, M.

Marty, Y.: vide Ducos, J.

Masukawa, T.: vide Moraes-Ruehsen, Maria D.

McInroy, R. A.: Exfoliative Scheidenzytologie. Überlegungen eines Zyto-Histo-

Pathologen 81

Meeker, C. I.: vide Watanabe, M.

Melander, S.: Listeriainfektion in der Gravídität vom geburtshilflichen Stand-

punkt 125

Mehbod, H.: vide Brest, A. N.

Merrill, J. A.: Untersuchungen über die Strahlenempfindlichkeit bei der

Behandlung des Kollumkarzinoms 98

Milani-Comparetti, M.: vide Gedda, L. Mirgone, O. R.: vide Blank, B.

VI

Inhaltsverzeichnis

Mitglieder der Bradford-Gruppe des College of General Practitioners: Ein Überblick über 100 Fälle von frühzeitiger Entlassung nach der Geburt 132

Montalegre, J.: vide Ducos, J.

Moore, J. G.; Wells, R. G. und Morton, D. G.: Die Behandlung des Ober-

flächenkarzinoms der Zervix in der Schwangerschaft $\quad 100$

Moraes-Ruehsen, Maria D. und Masukawa, T.: Abstrich aus Scheidenspülung

in der Hormonzytologie

91

Morton, D. G.: vide Moore, J. G.

Onesti, G.: vide Brest, A. N.

Palisa, V.; Jindra, J.; Tomecková, S. und Kalárová, J.: Die Problematik der

Gelbsuchtformen in der Schwangerschaft 117

Parks, A. G.: Die Hämorrhoidektomíe 228

Perez-Vitoria, C.: Leibschmerzen endokrinen Ursprungs 276

Phillips, S. F. und Edwards, D. A. W.: Untersuchungen über den Mechanis-

mus der Defäkation und der Stuhlkontinenz 152

Posner, N. A.: vide Scharfman, E.

Richards, I. D. G. und Lowe, C. R.: Veränderungen in der Totgeburtenrate in

England und Wales 135

Richart, R. M. und Vaillant, H. W.: Abstrich aus Scheidenspülung. Falsch-

negative Resultate bei Kranken mit Zervixkrebs 88

Robertson, J. G.: Zwillingsschwangerschaft: Einfluß der Frühhospitalisation auf die kindliche Überlebensrate 12

Ruiz, L. M.: Scheídenzytologie während der Entbindung

Salmon, Ch. : Unter welchen Umständen und mit welchen Mitteln können 
hämolytische Transfusionszwischenfälle in der Chirurgie verhindert wer-

den? $\quad 35$

Scharfman, E.; Posner, N. A. und Schocklander, J.: Ambulante Abrasio . . 95

Schirger, A. und Kavanaugh, G.: Geschwollene Beine bei alten Menschen .. 303

Schocklander, J.: vide Scharman, E.

Scholz, Magda: vide Galambos, L.

Schorno, O.: vide Kind, $\mathrm{H}$.

Schottenfeld, D. und Houde, R. W.: Veränderungen in der Krebsmorbidität

und -mortalität und daraus entstehende Schlußfolgerungen 299

Schreyer, H.: vide Kahr, E.

Seale, J. R.: Durch Geschlechtsverkehr übertragene Krankheiten in der Ehe . 225

Seigneurin, R.: vide Magnin, R.

Sims, E. A. H.: vide Watanabe, M.

Solomon, S.: vide Watanabe, M.

Spjut, H. J.: vide Kaufman, R. H.

Sprunt, D. H.: vide Lundin, F. E., Jr.

Starup, J.: Der Mechanismus der Ovulationshemmung bei der oralen Kontra-

zeption 21

Steczik, A.: vide Kiss, G.

Steingold, L.; Dunn, L.; Hawksworth, E. und Limb, L.: Der Einfluß der

Raumbelüftung auf den infektiösen Hospitalismus 8

Svanborg, A. und Vikrot, O.: Die Wirkung von Ostradiol und Progesteron

auf die Plasmalipide bei ovarektomierten Frauen $\quad 280$

Talaat, M.; Habib, Y. A.; Higazy, A. M.; Abdel Naby, S.; Malek, A. Y.

und Ibrahim, Z. A.: Der Einfluß von Sexualhormonen auf den Kohle-

hydratstoffwechsel der gesunden und der diabetischen Frau

Terrier, E.: vide Jaulmes, B. Thunell, G.: vide Bergman, P.

Inhaltsverzeichnis VII

Tizgard, J.-P. M.: Indikationen zur Sauerstofftherapie beim Neugeborenen 267

Tomecková, S.: vide Palisa, V.

Trine, G.: vide Josso, F.

Vaillant, H. W.: vide Richart, R. M.

Van den Driessche, A.: vide Lauweryns, J.-M.

Vikrot, O.: vide Svanborg, A.

Voigt, K. D.: vide Apostolakis, M.

Vorwort des Herausgebers der Zeitschrift « British Medical Journal»: Häusliche/

klinische Entbindung

6

Warsaw, E.: vide Harris, JhM.

Watanabe, M.; Meeker, C. I.; Gray, M. J.; Sims, E. A. H. und Solomon, S.:

Die Sekretionsrate des Aldosterons in der pathologischen Schwangerschaft 113 Wells, R. G.:

vide Moore, J. G. Williams, K. G.: vide Hutchinson, J. A.

World Health Organization: Intrauterine Antikonzeption 206

Zettergren, L.: vide Bergman, P.

Tagung der Tschechoslowakischen Gynäkologischen Gesellschaft, Prag, 3.-5. November 1966

Arenberger, M.: vide Dvorak, K.

Bártová, D.; Soska, J.; Srácek, J. und Uhmannová, V.: Veränderungen des 
Sexuallebens bei der Benützung intrauteriner Antikonzeption 186 Benesová, D.; Houdek, J. und Pelák, Z.: Das histologische Bild des Endometriums bei intrauteriner Antikonzeption $\quad 174$

Bouda, J.: vide Hradecký, L.

Cekan, Z. und Kincl, F. A.: Die protrahierte Wirkung von Antikonzeptionssteroiden im Experiment 203

Cepelák, J. und Havranek, F.: Einfluß der intrauterinen Antikonzeption auf Sexualaktivität und psychisches Verhalten der Frau 188

Cernoch, A.: vide Dvorak, K.

Dvorak, K. und Arenberger, M.: Sehstörungen nach Gabe von Antigest . . 199

Dvorak, K. und Cernoch, A.: Ein Vergleich zwischen der LIPPES-Schlinge und DANA $2 \quad 168$

Dvorak, K.: vide Havranek, F.

Dyková, H.: vide Havranek, F.

Dyková, H.: vide Havranek, F.

Havranek, F.; Dyková, H. und Tichý, M.: Durchgängigkeit der Tuben nach intrauteriner Antikonzeption 184

Havranek, F.; Dyková, H. und Tichý, M.: Veränderungen des Zervikal-

sekretes und des Postkoital-Testes nach Gaben von Antigest

Havranek, F.; Valenta, M. und Dvorak, K.: Zusammenhang zwischen Größe und Form von Intrauterinpessaren und Cavum uteri, untersucht mit der Methode der doppelten Röntgenbilddarstellung $\quad 169$

Havranek, F.: vide Cepelák, J.

Havranek, F.: vide Králová, A.

Heczko, P.: vide Procházka, J.

Hermanská, Z.: vide Misinger, I.

Houdek, J. und Pelák, Z.: Bakteriologische Befunde bei intrauteriner Anti

konzeption $\quad 180$

VIII

Inhaltsverzeichnis

Houdek, J. und Pelák, Z.: Der Einfluß der intrauterinen Antikonzeption auf

die Tubenpersitaltik 182

Houdek, J.: vide Benesová, D.

Hradecký, L.; Bouda, J. und Mleziva, J.: Intrauterine Antikonzeption und

terminale Strombahn im Endometrium

172

Kincl, F. A.: vide Cekan, Z. Kincl, F. A.: vide Rudel, H. W.

Kohoutek, M. und Vácha, K.: Schwangerschaft nach intrauteriner Antikon zeption 189

Králová, A. und Havranek, F.: Veränderungen der Endometriumzytologie nach intrauteriner Antikonzeption 178

Krobová, I.: vide Misinger, I.

Kucera, J.: vide Málek, J.

Málek, J. und Kucera, J.: Tagesrhythmus der vaginalen Temperaturen im

Menstrualzyklus und in der Schwangerschaft 141

Misinger, I.; Krobová, I. und Hermanská, Z.: Hämokoagulationsfaktoren bei der Antikonzeption mit Antigest 
Mleziva, J.: vide Hradecký, L.

Pelák, Z.: vide Benesová, D.

Pelák, Z.: vide Houdek, J.

Pelák, Z.: vide Houdek, J.

Progházka, J. und Heczko, P.: Veränderungen einiger Gerinnungsfaktoren

nach Gaben von Antigest 195

Rudel, H. W. und Kincl, F. A.: Schwangerschaftsverhütung durch kleine Pro-

gestagen-Gaben. Klinische Studie 202

Sochman, J. und Sterba, R.: Einfluß langzeitiger Antigest-Gaben auf die

Leberfunktionen 200

Soska, J.: vide Bártová, D.

SrÁcek, J.: Erfahrungen mit der Intrauterinantikonzeption. Ein zusammenfas-

sender Überblick 191

SrÁcek, J.: vide Bártová, D. Sterba, R.: vide Sochman, J. Tichý, M.: vide Havranek, F. Tichý, M.: vide Havranek, F.

Übersicht über weitere Referate, die an der Tagung gehalten wurden

Uhmannová, V.: vide Bártová, D.

Vácha, K.: vide Kohoutek, M.

Valenta, M.: vide Havranek, F.

Vojta, M.: Die heutigen Aufgaben der Antikonzeption

162

Vorwort 161 